\title{
Acute form of acne inversa in an 18-week pregnant patient: a case study
}

\author{
Katarzyna Plagens-Rotman ${ }^{1}$, Renata Przybylska², Katarzyna Gerke ${ }^{3}, K^{2} y s t y n a ~ J a r a c 2^{2}$, Justyna Serafinowska ${ }^{3}$, \\ Anna Sadowska-Przytocka ${ }^{3}$, Zygmunt Adamski ${ }^{3}$, Magdalena Czarnecka-Operacz ${ }^{3}$
}

${ }^{1}$ Department of Mother's and Child's Health, Poznan University of Medical Sciences, Poznan, Poland ${ }^{2}$ Department of Neurological Nursing, Poznan University of Medical Sciences, Poznan, Poland ${ }^{3}$ Department of Dermatology, Poznan University of Medical Sciences, Poznan, Poland

Adv Dermatol Allergol 2019; XXXVI (2): 242-246 DOI: https://doi.org/10.5114/ada.2018.79472

Acne inversa (hidradenitis suppurativa/acne inversa - HS/AL) is an acute chronic inflammatory disease occurring in the area of skin folds in the form of painful nodules with the tendency to form fistulae and scarring $[1,2]$. Changes cover gluteal and genital areas, groins, inguinal fossa and hairy skin on the head [2-4].

The prevalence rate of HS in Europe ranges between $1 \%$ and $4 \%$ and the disease is more common among post-pubescent men. Among children acne inverse is extremely rare, diagnosed before the age of 11 [5].

The aim of the study was to present a clinical case and methods of treatment of HS based on the case of an 18-week pregnant woman with an exacerbation of HS treated in 2017 at the Department and Clinic of Dermatology at the University of Medical Sciences in Poznan.

A 30-year-old pregnant woman was admitted to the Dermatology Clinic for treatment of HS. Skin lesions in the form of extensive abscesses and fistulae occur in both groin areas (Figure 1). The disease exacerbation had started at the beginning of the pregnancy. In the past, treatment with isotretinoin and surgical treatment were applied. On admission, the patient was in the $18^{\text {th }}$ week

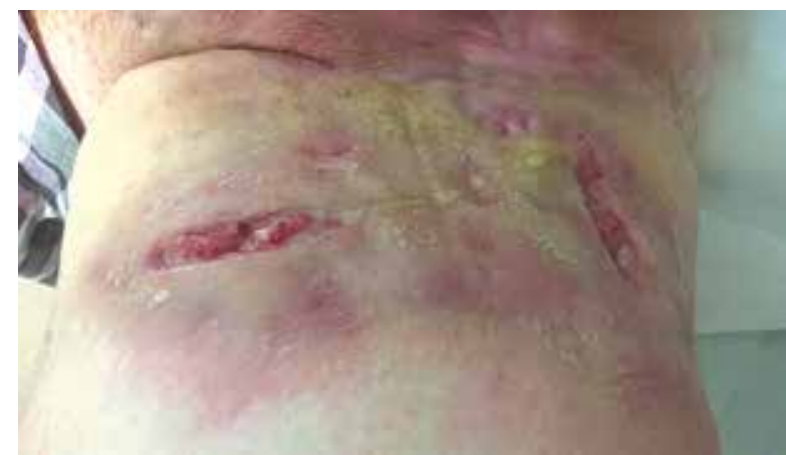

Figure 1. Skin changes in hidradenitis suppurativa of pregnancy. According to the opinion of the gynaecologist established 2 weeks before hospitalisation, the pregnancy was going well.

The lab tests results had revealed:

- leucocytosis (10.02.2017 - $12.26 \times 10^{3} / \mu \mathrm{l} ; 1.03 .2017$ $\left.9.40 \times 10^{3} / \mu \mathrm{l}\right)$;

- neutrocytosis $\left(10.02 .2017-9.02 \times 10^{3} / \mu l ; 1.03 .2017-\right.$ $\left.6.56 \times 10^{3} / \mu \mathrm{l}\right)$;

- reduced haemoglobin levels (10.02.216 - $8.6 \mathrm{~g} / \mathrm{dl}$; 1.03.2017 - $8.5 \mathrm{~g} / \mathrm{dl})$;

- high platelet count $\left(10.02 .2017-563 \times 10^{3} / \mu \mathrm{l} ; 1.03 .2017\right.$ $\left.-502 \times 10^{3} / \mu \mathrm{l}\right)$;

- elevated erythrocyte sedimentation rate (ESR) (10.02.2017 - $109 \mathrm{~mm} / \mathrm{h} ; 1.03 .2017-110 \mathrm{~mm} / \mathrm{h})$;

- Increased concentration of C-reactive protein (CRP) (10.02.2017 - 85.2 mg/l; 1.03.2017 - $23.8 \mathrm{mg} / \mathrm{l})$;

- Increased concentration of AST (10.02.2017 - $41 \mathrm{U} / \mathrm{I}$; 1.03.2017- $21 \mathrm{U} / \mathrm{I})$.

The patient was consulted gynaecologically. FHR (+), foetal movements (+), no signs of placenta abruption. Standard amount of the amniotic fluid. Vulva with scarring from the basic condition, hyperaemia mucosae. In internal examination: vaginal area formed, cervix smooth, uterine fundus $2 \mathrm{~cm}$ below the navel. Correct muscle tension. Recommendations: continued treatment of vaginal mycosis - one Clotrimazole pill inserted in the vagina in the evening. No contraindications to the treatment suggested.

Following the surgical consultation, the procedure of clearing the abscesses in general anaesthesia was planned. During hospitalization skin lesions were surgically removed - incision and drainage. After that, the negative pressure wound therapy was implemented using PICO dressings, with significant improvement of the clinical condition of the patient. Three changes of the dressing on the right side and two changes on the left

Address for correspondence: Katarzyna Plagens-Rotman MD, Department of Mother's and Child's Health, Poznan University of Medical Sciences, 41 Jackowskiego St, 60-512 Poznan, Poland, phone: +48 607-165-279, e-mail: plagens.rotman@gmail.com Received: 6.01.2018, accepted: 26.03.2018. 
side were done. On the day of discharge, another change of PICO dressings was planned.

The cream with hydrocortisone $1 \%$ and Ichthammol compresses were applied in topical treatment.

Erythromycin $600 \mathrm{mg}$ three times a day was applied. The patient was discharged from hospital in good general condition with the recommendation of further control of the condition in the dermatological and gynaecological outpatient clinic, regular blood pressure measurements and checking glucose concentration as well as restricting the consumption of monosaccharides and animal fats.

Causes of HS are not entirely known, but the genetic factors seem to play a role in causing the condition, in particular genes from the $\beta$-secretase complex - NCSTN, PSENEN and PSEN1 as well as environmental factors. Nowadays, it is estimated that $30-40 \%$ of patients report a family history of AL. It needs to be highlighted that the patients with gamma gene mutations suffer from the acute, extensive phenotype of the disease [6-8]. The $\gamma$-secretase complex includes four hydrophobic proteins: presenilin, nicastrin, as well as APH -1 and PEN - 2 . $\gamma$-secretase is one of subunits of the protease complex which cuts apart transmembrane proteins, and leads to appropriate processing of Notch protein. The $\beta$-secretase mutations have been the most important mutations in the loss of the function leading to the reduction in the activity of protease in the progress of hidradenitis suppurativa [8-10].

The exact mechanism of developing the condition remains unclear, however it is believed that initially it results from the occlusion of the upper part of hair follicles, which in turn leads to lymphohistiocytic follicular inflammation. The dysfunction of apocrine and eccrine sweat glands has appeared as a result of inflammatory reaction [11]. It is believed that some role is played by the interleukin-2 (IL-2) receptor. The proinflammatory cytokines have been isolated: IL-1 $\beta$, IL-10, tumour necrosis factor $\alpha$ (TNF- $\alpha$ ), TLR2 receptor (Toll-like receptors type 2) and anti-bacterial peptides IL-23 and IL-12, IL-10, IL-17. Additionally, a high expression of TLR2 receptors was observed [12-14].

The obesity and smoking have been established as risk factors for HS development. Kromann et al. [15] have indicated that a substantial body weight loss has protective influence on the development of the condition and its severity. It has been demonstrated that 2 years following the bariatric surgery, the body weight of the patients reduced by $35 \%$ can reduce the skin lesion intensity from 1.93 to 1.22 .

A study by Revuz et al. [16] has also demonstrated a clear correlation between HS and body mass index. The odds ratio of developing the condition for overweight and obese women is OR $=2.08$; $95 \% \mathrm{Cl}: 1.40-3.08$ and $\mathrm{OR}=$ 4.42; $95 \% \mathrm{Cl}: 2.82-6.93$. It is presumed that being overweight leads to mechanical irritation of skin folds, which leads to intensification of keratosis pilaris. For smoking women, the ratio is $\mathrm{OR}=12.55 ; 95 \% \mathrm{Cl}: 8.58-18.38$. Probably nicotine intensifies hyperkeratosis in hair follicles, inhibits apoptosis of neutrophils and leads to increased hydroxylation of oestrogens [15].

The nodules are most likely to form in the armpit area, in groins, around anus, on labia and buttocks. Painful, inflammatory nodules tend to break open and form fistulae. Multiple abscesses create extensive infiltration focuses with fistulae, which in turn leads to scar formation after the abscesses heal. The skin changes are painful. Additionally, they may be accompanied by general discomfort and increased body temperature $[2,17]$.

Differential diagnosis of acne inversa $[17,18]$ :

- skin manifestations in non-specific inflammatory bowel disease (including Lesniowski-Crohn's disease);

- inflammatory spondyloarthropathies;

- Staphylococcal infections;

- inflammation of hair follicles;

- perirectal abscesses, furuncles, fistulas;

- inguinal venereal lymphogranuloma;

- Bartholin's gland's inflammation;

- spinocellular carcinoma.

Acne inversa treatment in pregnant women [19-24]: - topical application of Clindamycin (1\%) as a first-line treatment at stage I/II HS;

- application of Clindamycin (600 mg)/Rifampicin (600 mg) at stage I/II HS or as a first-line treatment in therapy of II/III HS;

- application of dapsone as a late treatment of a mild or moderate form of HS;

- minimal surgical excision performed in local anaesthesia after the finished first trimester of pregnancy;

- use of Ibuprofen as a pain reliever during lactation;

- use of salicylates and non-steroidal anti-inflammatory drugs (NSAIDs), with the exception of acetaminophen, is not recommended;

- use of tramadol in post-operative care or in case of severe pain.

Nursing care in acne inversa:

- wash the skin not more than twice a day;

- avoid using coarse and skin irritating washcloths;

- use moisturizing agents which do not lead to creation of blackheads; it is recommended to use gel-or silicone-based agents (cyclomethicone, dimethicone);

- use cotton underwear;

- diet rich in protein, restricting the consumption of fats and carbohydrates;

- applying antioxidants, including vitamins, macro- and microelements;

The aim of the treatment is to minimize the symptoms and prevent development of new lesions.

The choice of the method of treatment is dependent on the level of development of the disease process.

Topical treatment of acne inversa - exfoliative agents - resorcinol (1,3 - dihydroxybenzene, $m$ - hydroxyphenol) is an antiseptic, antibacterial and keratolytic chemical 
peel. Using 15\% resorcinol among women with mild and moderate symptoms of HS reduces painful ailments and enables healing of skin manifestations [17, 25].

During the exacerbation of HS it is recommended to apply resorcinol twice a day and once a day during maintenance treatment [17].

It is important to note that toxicity of resorcinol was demonstrated after its transcutaneous absorption.

Remark: the use of Resinol Ointment is not recommended in pregnant women due to insufficient data on safety.

Antibiotic therapy of acne inversa - in the therapy of acne inversa it is recommended to use antibiotics from the tetracycline group: minocycline, ciprofloxacin, clindamycin, cephalosporins or topical antibiotic therapy (erythromycin, clindamycin). Additionally, cyclosporine A, sulphones and oestrogen and antiandrogen formulations are used (including cyproterone and finasteride) $[26,27]$. The most commonly recommended therapy is now clindamycin with rifampicin [28].

Clindamycin - belonging to the class of lincosamides it inhibits synthesis of bacteria proteins by blocking bacteria ribosomes and cell structures taking part in protein synthesis. It is also bacteriostatic and bactericidal in its broad spectrum of activity.

In mild and moderate symptoms of HS it is recommended to apply a balm containing clindamycin topically twice a day for 3 months [17].

Using the formulation by patients with patients suffering from diseases of the digestive tract (especially those having suffered large intestine inflammation) and those with liver conditions, myasthenia, Parkinson's disease should be carefully considered. Among patients with positive penicillin allergy tests, there is essential oedema and anaphylactic reactions may occur.

Remark: Clindamycin (1\%), metronidazole $(0.75 \%)$ and erythromycin (2\%) can be used twice daily in pregnant women (pregnancy category B) in case of the active changes of HS until the symptoms disappear.

Tetracycline - it inhibits synthesis of protein by binding to the 305 subunit of microbial ribosomes. It possesses bacteriostatic activity against Gram bacteria-Propionibacterium acnes, Gram-negative, Chlamydia, Rickettsia, Mycoplasma, Spirochaetaceae and some protozoans, but does not act against Enterococcus faecalis, Proteus spp., Serratia spp. and Pseudomonas aeruginosa.

It is recommended to use $500 \mathrm{~g}$ tetracycline twice a day for 4 months. Patients must be observed due to potential acute liver failure. It is not recommended to prescribe tetracycline to pregnant women and children under 9 years old as the antibiotic may lead to permanent teeth discolouring and leads to tooth enamel hypoplasia [17].

Remark: tetracyclines are contraindicated in pregnant and breast-feeding women (pregnancy category D);
Clindamycin - Rifampicin inhibits bacterial DNAdependent RNA synthesis by inhibiting bacterial DNA and growth of Gram-positive bacteria (Staphylococcus aureus, Staphylococcus epidermidis) and Gram-negative bacteria (Neisseria meningitidis, Neisseria gonorrhoeae, Legionella spp., Haemophilus influenzae).

It is recommended to use $300 \mathrm{mg}$ clindamycin twice a day with $600 \mathrm{mg}$ rifampicin (in a single dose or twice a day in $300 \mathrm{mg}$ dose) for 10 weeks among patients with mild or moderate symptoms of HS $[19,28]$. In case of lack of improvement of the dermatological condition, other methods of treating HS should be considered.

When treating patients with rifampicin, special caution needs to be exercised with patients suffering from kidney or liver dysfunctions. In these cases it is recommended to use smaller doses and monitor liver activity by measuring ALT and AST. With pregnant or breastfeeding women it needs to be considered whether the advantages for the mother outweigh the potential risks for the child.

Rifampicin permeates to breastmilk but it does not cause any adverse effects in the breastfed infants (pregnancy category C), whereas clindamycin is considered safe for use in pregnancy (pregnancy category B).

Anti-inflammatory treatment:

Glucocorticoids (GCS) inhibit inflammatory processes by blocking synthesis of IL-1, IL-3, IL-4, IL-5, IL-6, IL-8, TNF- $\alpha$ and granulocyte-macrophage colony-stimulating factor (GM-CSF), are antiprolific by inhibiting DNA and collagen synthesis and immunosuppressive by inhibiting proliferation of lymphocytes $T$ and B, Langerhans cells and mastocytes.

When choosing GCS as a method of treatment of HS, many side effects resulting from inhibiting the synthesis of collagen, elastin, glycosaminoglycans and proliferation of keratinocytes and fibroblasts should be taken into account. The most important adverse effects are: epidermal and dermal thinning, diastema, telangiectasias, dermatitis perioralis type of skin changes (perioral inflammation), post steroid acne, bacterial, virus, parasite and/or fungal superinfections [17].

Sulfones (4-4'-diaminodiphenylsulfone) have antibacterial, antiparasitic and anti-inflammatory properties by inhibiting multinuclear leukocyte chemotaxis and activating the complement. It is recommended to use the therapy in 25-200 mg/day dose [29]. Increasing the dosage may be impossible because of side effects, i.e. nausea, stomach-ache, reduced appetite and vomiting, hepatotoxicity, haemolytic anaemia, peripheral neuropathy, bullous erythema multiforme, urticaria, erythema nodosum, morbilliform or scarlet-fever-form rash. Another possible adverse effect among patients receiving dapsone is developing drug-induced aseptic meningitis.

Before initiating the therapy it is recommended to have complete blood count, liver function tests (ALT, AST, bilirubin, GGT) and kidney function test (creatinine), gen- 
eral urine test, serological tests for hepatitis, marking activity of glucose-6-phosphate dehydrogenase [30].

Cyclosporine A (CyA) belongs to calcineurin inhibitors selectively affecting T lymphocytes. It has strong immunosuppressive properties. Cyclosporin A-cyclophilin complex prevents from phosphorylation of nuclear factor of activated T-cells, blocking the transcription of interleukin 2 [31].

Data about dosage and length of treatment are limited, but successful results of treatment are achieved with the dosages of $2.5-5 \mathrm{mg} / \mathrm{kg}$ a day [32].

The contraindications to cyclosporine are untreated hypertension, kidney failure, acute infection and patients with diagnosed malicious carcinoma (except for basalcell carcinoma). Moreover, extra caution is recommended with pregnant patients, during lactation, and among patients with acute liver failure, primary or secondary immunodeficiency, diabetes, obesity and old age (> 65 years old).

Remark: No evidence for teratogenicity of cyclosporine $A$ in the foetus was found.

Biological treatment:

- Inhibitors of TNF- $\alpha$ stimulate neutrophils, dendritic cells and macrophages [32]:

- Etanercept - $5 \mathrm{mg} / \mathrm{kg}$ body mass in weeks 0, 2 and 6 , and next for 8 weeks administered intravenously;

- Infliximab - $2 \times 50 \mathrm{mg} /$ week (for the first 3 months) or $2 \times 25 \mathrm{mg} /$ week or $1 \times 50 \mathrm{mg} /$ week administered subcutaneously;

- Adalimumab - $80 \mathrm{mg}$ in the $1^{\text {st }}$ week, $40 \mathrm{mg}$ in the $2^{\text {nd }}$ week, and next $40 \mathrm{mg}$ every 2 weeks;

- Ustekinumab - it blocks interleukin IL-12 and IL-23 and maturation of virgin lymphocytes changing into lymphocytes T. $17-45 \mathrm{mg}$ (for patients $<100 \mathrm{~kg}$ ) or $90 \mathrm{mg}$ (for patients $>100 \mathrm{~kg}$ ) in weeks 0 and 4, and next every 12 weeks administered subcutaneously.

Remark: in case of biological treatment, the safety of its use during pregnancy and breastfeeding has not been established.

Retinoids - synthetic aromatic derivatives of vitamin A that regulate cell proliferation and differentiation of keratinocytes and restrict inflammation. Indication for using them is the acute form of acne: acne conglobata, acne inversa, acne fulminans, and acne papulopustulosa [17]: - Acitretin - in dosage of $0.3-0.5 \mathrm{mg} / \mathrm{kg}$;

- Isotretinoin - synthetic stereoisomer of all-trans retinoic acid (13-cis-retinoic acid);

- Etretinate - in dosage of 0.6-1.0 mg/kg;

- According to research, acitretin is most efficient.

Remark: what needs to be emphasized is the teratogenic side effects of using retinoids. For in maternal age women, contraception during retinoid treatment is necessary, also for the period of 1 month up to 2 years since completing treatment (depending on the medication used).

Negative pressure would therapy (NPWT) dressings create moist healing environment, protect from harmful external factors, reduce swelling and increase vascular perfusion. Additionally, they remove the fluid from the wound, and thus reduce the risk in the case of bacterial infection [33-35].

Contraindications to using negative pressure wound therapy include:

- diagnosed carcinoma;

- necrotic tissues around the wound;

- untreated osteomyelitis;

- extraintestinal fistulae;

- exposed vessels, nerves, bones or organs.

Before introducing negative pressure would therapy the wound has to be cleaned of the necrotic tissues and its structures should be covered with fascia or nonadherent material, e.g. silicone dressing protecting the tissues from growing into the polyurethane foam. Moreover, with infections it is important to apply topical and systematic treatment with antibacterial, antifungal and antibiotic dressings [35]. Treating concomitant diseases consists in eliminating the factors disrupting the process of wound healing by controlling metabolic dysfunctions in diabetes or malnutrition.

Complications of acne inversa: scars; infections; anaemia; nephrotic syndrome; arthropathies; secondary lymphatic swelling (of scrotum or vulva); fistulae of rectum, vagina, urethra and urinary bladder as consequences of inflammation; SAPHO syndrome - synovitis, acne, hyperostosis and osteitis; Pyoderma gangrenosum and secondary amyloidosis affecting the kidneys and heart; interstitial keratitis leading to the perforation of cornea; spinocellular carcinoma.

Acne inversa poses a great therapeutic problem in dermatology, gynaecology and surgery. Moreover, it is a source of suffering and frustration in patients. Depression and fear are most significant among patients with the disease affecting the perianal area.

There is no doubt that nursing care, modification of lifestyle and patient's education play significant roles in the treatment of HS. The medical treatment of the conditions includes not only antibiotics but also immunosuppressive medications. Surgical treatment should be used after applying all remaining methods of treatment available.

\section{Conflict of interest}

The authors declare no conflict of interest.

\section{References}

1. Scheinfeld N. Hidradenitis suppurativa: a practical review of possible medical treatments based on over 350 hidradenitis patients. Dermatol Online J 2013; 19: 1.

2. Witmanowski H, Szychta P, Stępniewski S, et al. Acne inversa goes an extra mile than hidradenitis suppurativa. Adv Dermatol Alergol 2013; 30: 255-60. 
3. Bergler-Czop B, Hadasik K, Brzezińska-Wcisło L. Acne inversa: difficulties in diagnostics and therapy. Adv Dermatol Allergol 2015; 32: 296-301.

4. Jemec GB. Clinical practice. Hidradenitis suppurativa. N Engl J Med 2012; 366: 158-64.

5. Randhawa HK, Hamilton J, Pope E. Finasteride for the treatment of hidradenitis suppurativa in children and adolescents. JAMA Dermatol 2013; 149: 732-5.

6. Pink AE, Simpson MA, Desai N, et al. gamma-Secretase mutations in hidradenitis suppurativa: new insights into disease pathogenesis. J Invest Dermatol 2013; 133: 601-7.

7. Pink AE, Simpson MA, Desai N, et al. Mutations in the gamma-secretase genes NCSTN, PSENEN, and PSEN1 underlie rare forms of hidradenitis suppurativa (acne inversa). J Invest Dermatol 2012; 132: 2459-61.

8. Wang B, Yang W, Wen W, et al. Gamma-secretase gene mutations in familial acne inversa. Science 2010; 330: 1065.

9. Ingram JR, Piguet V. Phenotypic heterogeneity in hidradenitis suppurativa (acne inversa): classification is an essential step toward personalized therapy. J Invest Dermatol 2013; 133: 1453-6.

10. van der Zee HH, Prens EP. Preliminary findings suggest hidradenitis suppurativa may be due to defective follicular support. Br J Dermatol 2013; 168: 926-7.

11. von Laffert M, Helmbold P, Wohlrab J, et al. Hidradenitis suppurativa (acne inversa): early inflammatory events at terminal follicles and at interfollicular epidermis. Exp Dermatol 2010; 19: 533-7.

12. Schlapbach C, Hanni T, Yawalkar N, et al. Expression of the IL-23/Th17 pathway in lesions of hidradenitis suppurativa. J Am Acad Dermatol 2011; 65: 790-8.

13. Danby FW, Jemec GB, Marsch WC, von Laffert M. Preliminary findings suggest hidradenitis suppurativa may be due to defective follicular support. Br I Dermatol 2013; 168: 1034-9.

14. Wollina $U$, Koch A, Heinig B, et al. Acne inversa (Hidradenitis suppurativa): a review with a focus on pathogenesis and treatment. Indian Dermatol Online J 2013; 4: 2-11.

15. Kromann CB, Ibler KS, Kristiansen VB, Jemec GB. The influence of body weight on the prevalence and severity of hidradenitis suppurativa. Acta Derm Venereol 2014; 94: 553-7.

16. Revuz JE, Canoui-Poitrine F, Wolkenstein P, et al. Prevalence and factors associated with hidradenitis suppurativa: results from two case-control studies. J Am Acad Dermatol 2008; 59: 596-601.

17. Zouboulis CC, Desai N, Emtestam L, et al. European S1 guideline for the treatment of hidradenitis suppurativa/ acne inversa. J Eur Acad Dermatol Venereol 2015; 29: 619-44.

18. van der Zee HH, Prens EP. Failure of anti-interleukin-1 therapy in severe hidradenitis suppurativa: a case report. Dermatology 2013; 226: 97-100.

19. Gulliver W, Zouboulis CC, Prens E, et al. Evidence-based approach to the treatment of hidradenitis suppurativa/acne inversa, based on the European guidelines for hidradenitis suppurativa. Rev Endocr Metab Disord 2016; 17: 343-51.

20. Tyler KH, Zirwas MJ. Pregnancy and dermatologic therapy. J Am Acad Dermatol 2013; 68: 663-71.

21. Braunstein I, Werth V. Treatment of dermatologic connective tissue disease and autoimmune blistering disorders in pregnancy. Dermatol Ther 2013; 26: 354-63.

22. Deckers IE, Prens EP. An update on medical treatment options for hidradenitis suppurativa. Drugs 2016; 76: 215-29.

23. American College of O, Gynecologists. ACOG Committee opinion no. 548: weight gain during pregnancy. Obstet Gynecol 2013; 121: 210-2.
24. Verdolini R, Clayton N, Smith A, et al. Metformin for the treatment of hidradenitis suppurativa: a little help along the way. J Eur Acad Dermatol Venereol 2013; 27: 1101-8.

25. Boer J, Jemec GB. Resorcinol peels as a possible self-treatment of painfulnodules in hidradenitis suppurativa. Clin Exp Dermatol 2010; 35: 36-40.

26. van Rappard DC, Leenarts MF, Meijerink-van 't Oost L, et al. SComparing treatment outcome of infliximab and adalimumab in patients with severe hidradenitis suppurativa. J Dermatolog Treat 2012; 23: 284-9.

27. Amano M, Grant A, Kerdel FA. A prospective open-label clinical trial of adalimumab for the treatment of hidradenitis suppurativa. Int I Dermatol 2010; 49: 950-5.

28. Gener G, Canoui-Poitrine F, Revuz JE, et al. Combination therapy with clindamycin and rifampicin for hidradenitis suppurativa: a series of 116 consecutive patients. Dermatology 2009; 219: 148-54.

29. Kaur MR, Lewis HM. Hidradenitis suppurativa treated with dapsone: a case series of five patients. I Dermatolog Treat 2006; 17: 211-3.

30. Żychowska M, Batycka-Baran A, Szepietowski J, Baran W. Dapsone - mechanism of action, safety of use and the role in the treatment of bullous pemphigoid according to current recommendations. Przegl Dermatol 2016; 103: 176-84.

31. Amor KT, Caitriona R, Menter A. The use of cyclosporine in dermatology: part I. J Am Acad Dermatol 2010; 63: 925-46.

32. Szepietowski J, Adamski Z, Chodorowska G, et al. Treatment of psoriasis vulgaris: guidelines of experts of the Polish Dermatological Society. Part II: moderate to severe psoriasis. Przegl Dermatol 2014; 101: 455-72.

33. Thakrar BT, Robinson NJ. Isotretinoin use and the risk of inflammatory bowel disease. Am J Gastroenterol 2011; 106: 1000-3.

34. Addor FA, Schalka S. Acne in adult women: epidemiological, diagnostic and therapeutic aspects. An Bras Dermatol 2010; 85: 789-95.

35. Parrado R, Cadena M, Vergara A, et al. The role of negative pressure wound therapy in the management of hidradenitis suppurativa: a case report and literature review. Int Wound J 2017; 14: 35-9. 